\title{
Simulation Analysis of Temperature Controlling for Resistance Furnace Based on Hybrid Particle Swarm Optimization
}

\author{
Yuqin Yao ${ }^{1, a}$, Shiyu Hu ${ }^{2}$ \\ ${ }^{1}$ Chengdu University of Information Technology, Chengdu, 610225, China \\ ${ }^{2}$ Sichuan Meiguhe hydropower development company, LTD Chengdu, 611130 , China \\ ${ }^{a}$ Email: yyq@cuit.edu.cn
}

Key words: Hybrid Particle Swarm Optimization; resistance furnace; temperature; control; control theory and engineering; intelligent control theory

\begin{abstract}
The temperature is an important parameter for resistance furnace among the industries. Its nonlinear characteristics makes it is difficult to be controlled, therefore it is necessary to find out an effective method to get the resistance furnace temperature under control. The hybrid particle swarm algorithm and PID controller are combined to design the temperature controlling system of resistance furnace. Based on real situation of resistance furnace temperature control, the mathematical model of hybrid particle swarm algorithm is established. The chemotaxis, dispersion and reproduction of bacteria are introduced into the hybrid particle warm algorithm. According to the controlling theory, the basic procedure of hybrid PSO algorithm is designed and a temperature control system used for resistance furnace is developed too. The performance of control system is tested through simulation analysis and the simulation results are compared with the onsite data collected. The results of simulation show that the simulation value is close to the measured value, therefore, good resistance furnace temperature control result is obtained.
\end{abstract}

\section{Introduction}

Resistance furnace is widely used among the industries, such as chemical and metallurgy. With its significant status in the industrial production, the operation performance of the resistance furnace always decides the economic benefit of the whole industrial production. Therefore, make sure effective control of the resistance furnace is necessary. Temperature of the resistance furnace is the primary control indicator. However, there are certain difficult to get the resistance furnace temperature under control ${ }^{[1]}$. The main reasons are due to the changes of the resistance furnace temperature are time varying and non-linear. Therefore, in order to improve the temperature control result and ensure successful industrial production, a valid mechanism need to be applied.

Controlling models of resistance furnace temperature show complexity and solving of the models is also complicated procedures; therefore, controlling the temperature via intelligent computing will be a wise option. Existing intelligent computing methods are vary, such as ACO, artificial neural network, GA and PSO etc. But there are downsides for the above algorithms like low precision and computational efficiency. They are fairly easy to trap in local optimum when dealing with non-linear issues ${ }^{[2]}$. As a result, find a more effective algorithm is necessary. Hybrid Particle Swarm Optimization improves both the computational precision and efficiency when dealing with non-linear issues. Therefore, it is able to improve the resistance furnace temperature control result.

\section{2 . Fundamental principle of resistance furnace temperature control}

The temperature of the resistance furnace is subject to continuous change with the process requirements during the industrial production, which leads to the changes made for the resistance furnace temperature control method. In normal situation, resistance furnace temperature control 
includes two stages, the dynamic stage and the steady stage. The purpose at the dynamic control stage is to get the heat storage capacity under effective control. This is to ensure the resistance furnace temperature meet the basic requirement of the industrial production ${ }^{[3]}$. The effect of the steady control stage is to ensure a stable balanced status is maintained between the heating capacity and the heat dissipating capacity of the resistance furnace and then further to keep consistent resistance furnace temperature. The interaction of the static characterization of resistance furnace temperature and the heating voltage can be used at the steady control stage when controlling the resistance furnace temperature. Based on the resistance furnace temperature control requirements, PID technology is a common option to control the resistance furnace temperature. It based on the dynamic variation to select the input voltage, which is in accordance with the set value. The control efficiency will increase if it is controlled relatively fast. But it is highly likely cause numerical oscillation and finally leads to overshoot. To prevent this defect, the temperature control speed has to be slow down. When the resistance furnace temperature is change, the static gain of the resistance furnace temperature will change too. If the set temperature of the resistance furnace changes in relatively wide range, then the common PID control technology is not able to ensure the best temperature control result. So, PID technology that is based on hybrid particle swarm optimization can be utilized to control the resistance furnace temperature.

\section{3 . Mathematical model of the hybrid particle swarm optimization}

In 1995, American scholars Kenned and Eberhart introduced the creative particle swarm optimization. The basic idea of particle swarm optimization is to design the algorithm in accordance with the social behavior rule of fish schooling or bird flocking when they are finding food, and hence achieve global optimization ${ }^{[4]}$. Various optimization problems can be solved effectively through particle swarm optimization and it is able to turn all the problems into a volume less and massless particle which runs in the search space. All the particles have their corresponding fitness; the fitness value can be defined by using the optimized objective function. In addition, movement distances and directions of different particles can always be characterized by the corresponding speed. It is able to use the individual and swarm movement experience to define the movement speed and direction of the particle. In each iteration, particle can adjust its own movement speed and direction according the two "extremum" it looking for, which is individual extremum and global extremum. The individual extremum is the optimum solution for the particle, while the global extremum is the optimum solution for all particles. At the stage to obtain the individual extremum and the global extremum, speed and position of the particle can be determined through the formula as follow :

$$
v_{i}^{t}=\lambda \cdot V_{i}^{t-1}+c_{a} \cdot \operatorname{rand}() \cdot\left(p_{i}^{t-1}-x_{i}^{t-1}\right)+c_{b} \cdot \operatorname{rand}() \cdot\left(p_{d}^{t-1}-x_{i}^{t-1}\right)
$$

$x_{i}^{t-1}=x_{i}^{t-1}+v_{i}^{t-1}$

Within the formula, the $x$ represents current position of the particle. The $v$ represents the current movement speed of the particle. The $p_{i}$ represents the particle's extremum. The $p_{d}$ represents the global extremum of the particle swarm. The $c_{a}$ and $c_{b}$ represent the weighting factor of every particle's individual extremum and global extremum respectively, with the change interval $(0,1)$.The rand represent the random function between 0 and 1 . The $\lambda$ represents the inertia weighting factor. The space of particle optimization can be expanded and the global optimum value can be obtained through the inertia weighting factor. If the $\lambda$ is relatively big, it reduces the probability that the algorithm traps in minimal value. If $\lambda$ is relatively small, then the algorithm is easily enter into convergence state. Therefore, corresponding inertia weighting factor adjustment algorithm can be designed to continuous achieve inertia weighting factor linearly decrease in continuous iterations. According to the actual needs of the global search, if the particle can be ensured with better level of optimization in the initial optimization stage, then it may have better development ability at the end of optimization ${ }^{[5]}$. In this way, the algorithm will reach better convergence efficiency. The model of inertia weighting factor adjustment algorithm is as follow : 


\section{$\lambda=\lambda-\operatorname{iter}\left(\lambda_{\max }-\lambda_{\min }\right) /$ iter $_{\max }$}

Within the formula, the iter represents the iteration number of the algorithm. The iter $r_{\max }$ represents the maximum iteration number in the optimization stage. The $\lambda_{\max }$ represents the maximum inertia weighting factor and the $\lambda_{\min }$ represents the minimum inertia weighting factor. Whereby, the change interval for $\lambda_{\max }$ is $\{1.2,1.0,0.80\}$, while the change interval for $\lambda_{\min }$ is $\{0,0.3,0.5\}$.

When optimizing the particle swarm, the particle swarm keeps continuous iterative computing in the optimization space. If certain particle locates the optimum position, then other particles in the swarm will approach to this particle. When this position becomes the local optimum, the particle swarm will no longer be able to carry out continuous iterative computing in the whole optimization space. Under such situation, the particle swarm will gathering at the local optimum position and causes premature convergence, hence traps in local optimum. In order to avoid the above downsides, the particle swarm algorithm need to be improved by integrating the bacterial foraging algorithm. In 2002, K.M.Passino introduced the creative bacterial foraging optimization algorithm. The theory foundation of this algorithm is bionics, the idea is to design the algorithm in accordance with the behaviors when the Escherichia coli foraging in the intestine. The bacteria colonies forage in a continuous way and are able to realize the optimization. The final solution for the optimization is fitness. There are many advantages for bacterial foraging optimization algorithm, it is able to carry out chemo taxis, dispersion and reproduction computing. Hybrid particle swarm optimization algorithm is the outcome by combining the particle swarm optimization and the bacterial foraging optimization, the computing model is as follow :

$$
J(m, n, Q, S)=F(:, m, n, Q, S)
$$

Within the model, the $m$ represents the number of bacteria. The $n$ represents the chemokine. The $Q$ represents reproduction factor. The $S$ represents the dispersion coefficient. The $F$ represents the fitness function of the bacteria.

Within the optimization space, chemo taxis, dispersion and reproduction can ensure the bacteria updated continuously. The particles' movement positions and movement speed can be adjusted in accordance with the fitness function. The individual extremum and global extremum can be computed with the formula as follow :

$$
\begin{aligned}
& \text { simple_location }(:, m, n)=\text { present_locaiton }(:, m, \operatorname{index}(m,:)) \\
& \text { entity_location }(:, m, n)=\text { present_locaiton }(:, L, \operatorname{index}(L,:))
\end{aligned}
$$

Based on formula (5) and (6), the particle is able to carry out iterative computing in the optimization space. When global optimum solution is obtained, iterative computing is ceased.

The particle swarm's global search level will be improved through bacterial foraging optimization, and it is further to avoid premature convergence. The particle adjustment is achieved through bacteria update, which prevents the premature convergence that happens along with the changes in the fitness function in the conventional particle swarm optimization. By integrating bacteria chemotaxis, dispersion and reproduction computing into the particle swarm optimization, preventing premature convergence problem, avoiding the algorithm trap in local optimum, the movement speed and movement position are adjusted during the continuous particle adjustment process, the global optimum solution will be finally achieved.

Steps of hybrid particle swarm algorithm are as follow :

Particle swarm initialization operation Set the particle swarm scale, particle movement position and movement speed, set necessary parameters for other algorithms;

(2) Bacteria initialization operation Set the bacteria colonies scale and position, set the necessary step length for the bacteria colonies chemo taxis and reproduction processes, set the dispersion factor and reproduction factor;

(3) Set vector quantity of the particle movement position as the parameter of the PID controller and solve the corresponding fitness, initialize the individual extremum and global extremum;

(4)Covert the particle, find the optimum position for the particle and adjust the particle swarm 
fitness value, as well as the individual extremum and global extremum;

(5) Analyze the adjusted particle swarm fitness to see if the result improved. If improvement happens, then move to the next operation, if not, go back to step (4);

(6) Discriminate if the particle reaches the chemo taxis steps. If yes, then proceed the particle reproduction and dispersion. If not, go back to step (4);

(7) If bacteria are highly consumed, then dispersion operation need to be carried out based on the corresponding probability to update the bacteria colony, otherwise, go back to step (4);

(8) When maximum iteration number is meet, the algorithm is completed, otherwise, go back to step (4).

\section{Resistance furnace temperature control system design based on hybrid particle swarm optimization algorithm}

Combine hybrid particle swarm optimization algorithm with PID controller to design the resistance furnace temperature control system, model for computing system error and controlled quantity is as follow:

$$
\begin{aligned}
& \Delta u(i)=K_{p}[e(i)-e(i-1)]+K_{i} e(i)+K_{d}[e(i)-2 e(i-1)+e(i-2)] \\
& u(i)=u(i-1)+\Delta u(i)
\end{aligned}
$$

According to the basic principle of PID controller based on hybrid particle swarm optimization algorithm, adjust the PID controller basic parameters $K_{p}, K_{i}$ and $K_{d}$ in the sampling period through hybrid particle swarm optimization algorithm. Proceed the iteration computing according to time error of $k, k-1$ and $k-2$. Particle controlled quantity and fitness will be computed each time and select the particle with individual extremum and particle with global extremum by the fitness. The optimum particles selected after the iteration computing will be chosen as the $k+1$ time PID controller basic parameters. Resistance furnace temperature PID control system based on hybrid particle swarm optimization algorithm is shown in Figure 1.

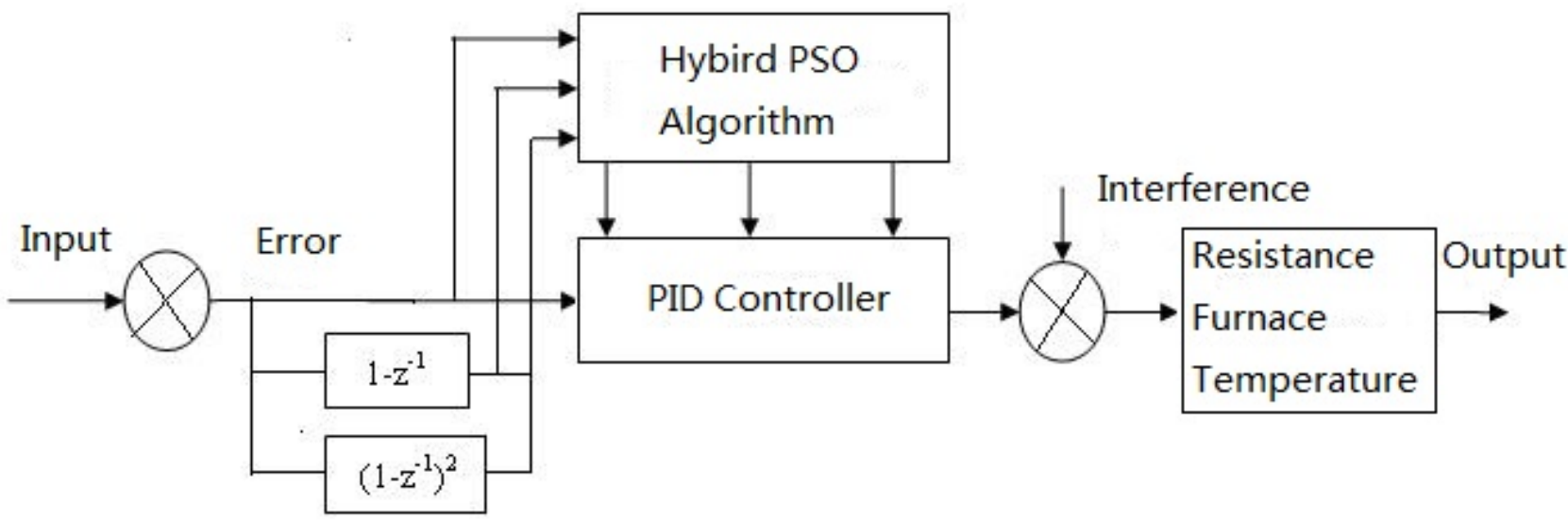

Figure 1. Resistance furnace temperature control system based on hybrid PSO algorithm

\section{Simulation research on resistance furnace temperature control system}

Parameters of hybrid PSO algorithm are set as follow: scale of particle swarm is 90, particles in the swarm are set as $\left[K_{p}, K_{i}, K_{d}\right], c_{a}=3.0, c_{b}=2.5, \lambda_{\max }=0.90, \lambda_{\text {min }}=0.60, \lambda_{1}=0.99$, $\lambda_{2}=0.001, \lambda_{3}=1.0, \lambda_{4}=100$. Sample step length is set as $0.001 \mathrm{~s}$; the maximum iteration number is 100 times. Transfer function of resistance furnace temperature control is as follow :

$$
G(s)=\frac{5 s+6}{(0.02 s+1)(0.04 s+1)(0.40 s+1)(6 s+1)}
$$

In order to verify advantages of the resistance furnace temperature control system based on hybrid PSO algorithm, the conventional resistance furnace temperature PID control system and the 
modified resistance furnace temperature PID control system are used to proceed the simulation analyses respectively. Windows2003 system, 512M RAM, CPU2.0 and dual core are chosen for the simulation platform. Simulation program is programmed with MATAB7.0 to proceed the simulation analyze. Simulation result is shown in Figure 2 Figure 2 shows the simulation curve of the resistance furnace temperature control. As can be seen from Figure 2, compared to the conventional resistance furnace temperature control system, the resistance furnace control system based on hybrid PSO algorithm has lots of advantages, such as shorter response time, smaller overshoot. Therefore, it has better control accuracy.

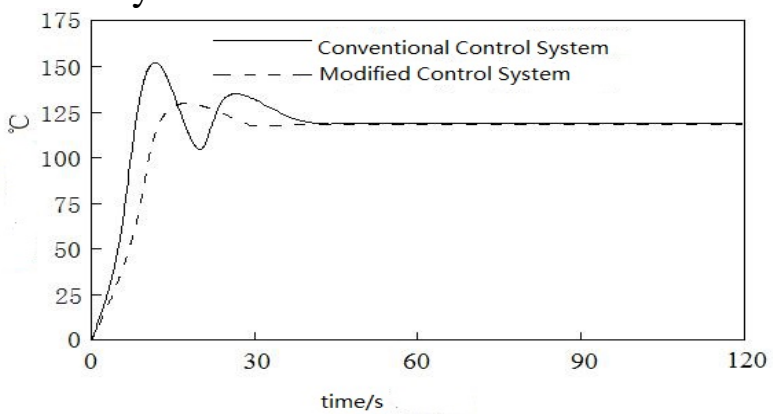

Figure 2. Comparison results of the two resistance furnace temperature control systems

In order to verify actual performance of ACO, on site resistance furnace temperature data is collected at an enterprise. ACO is applied to control the resistance temperature under the same operation condition. Comparison analyze is performed, the results are shown in Figure 3.

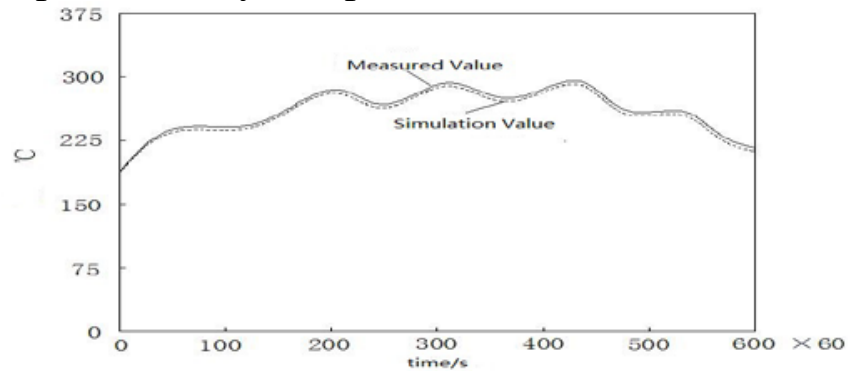

Figure 3. Measured value and simulation value of the resistance furnace temperature control

As can be seen from Figure 3, measured value is closer to the actual temperature change if controlled by the AOC. It thus shows that this algorithm has better applicability and is applicable to the actual control of the resistance furnace temperature.

\section{Conclusions}

Resistance furnace temperature is a main parameter. By controlling this parameter, the industrial production stability will be ensured. Due to time-lag and relative bigger non-linear features of the changing temperature, hybrid PSO algorithm and PID controller are combined together to design the resistance furnace temperature control system. And then the simulation analyze is carried out. The result shows that the modified resistance furnace temperature control system has better control accuracy and is able to reduce the system complexity.

\section{Reference}

[1] ShenXu. Rubber wheel curing furnace temperature control system based on PLC, the design and implementation [J]. Electrical applications, 2013, 32 (2) : 56-58.

[2] Wang Xiaoyan Zhou Zhiwen. Fuzzy control in the application of vacuum heat treatment furnace temperature control [J]. Automation and instrumentation, 2013, (1) : 84-85.

[3] Meng Jie, Chen Qing zhang, zhang kai. Automotive suspension PID control based on particle swarm algorithm simulation [J]. Computer simulation, 2013, 30 (4) : 155-158.

[4] Du Wenzheng Xie Zheng, TongGuoLin. Bridge crane based on particle swarm optimization 
algorithm control parameters optimization [J]. Journal of computer measurement and control, 2013, $21(2): 371-373$.

[5] D. Johnson, C. Perkins, J. Arkko. Mobility Support in IPv6. RFC 3775. 2003 